\title{
Effects of Paired Housing and Acute d-Amphetamine on Delay Discounting in Lewis and Fischer 344 rats
}

\author{
Marissa Turturici
}

Follow this and additional works at: https://researchrepository.wvu.edu/etd

\section{Recommended Citation}

Turturici, Marissa, "Effects of Paired Housing and Acute d-Amphetamine on Delay Discounting in Lewis and Fischer 344 rats" (2014). Graduate Theses, Dissertations, and Problem Reports. 6841.

https://researchrepository.wvu.edu/etd/6841

This Thesis is protected by copyright and/or related rights. It has been brought to you by the The Research Repository @ WVU with permission from the rights-holder(s). You are free to use this Thesis in any way that is permitted by the copyright and related rights legislation that applies to your use. For other uses you must obtain permission from the rights-holder(s) directly, unless additional rights are indicated by a Creative Commons license in the record and/ or on the work itself. This Thesis has been accepted for inclusion in WVU Graduate Theses, Dissertations, and Problem Reports collection by an authorized administrator of The Research Repository @ WVU. For more information, please contact researchrepository@mail.wvu.edu. 
Effects of Paired Housing and Acute $d$-Amphetamine on Delay Discounting in Lewis and Fischer 344 rats

\author{
Marissa Turturici
}

Thesis submitted to the Eberly College of Arts and Sciences

at West Virginia University

in partial fulfillment of the requirements for the degree of

Master of Science in Psychology

Committee Members

Karen G. Anderson, Ph.D., Chair

Elizabeth Kyonka, Ph.D.

Melissa Blank, Ph.D.

Department of Psychology

Morgantown, West Virginia

2014

Keywords: $d$-amphetamine, delay discounting, Lewis, Fischer 344, rat, paired housing, impulsive choice

Copyright 2014

Marissa Turturici

All Rights Reserved 


\begin{abstract}
Effects of Paired Housing and Acute $d$-Amphetamine Administration on Delay Discounting in
\end{abstract}

Lewis and Fischer 344 rats

\title{
Marissa Turturici
}

Lewis (LEW) and Fischer 344 (F344) rats are two genetically different strains that differ in impulsive choices made in a delay-discounting task. An operational definition of impulsive choice is a choice of a smaller-sooner reinforcer over a larger-later reinforcer. Research using LEW and F344 rats has housed rats singly. Studies have shown that paired housing influences impulsive choice, but it is not known if paired housing affects impulsive choice in the LEW and F344 strains specifically. The present study compared impulsive choice in pair-housed LEW and F344 rats to archival data from singly housed LEW and F344 rats. Impulsive choices were measured at baseline and after $d$-amphetamine ( $d$-AMP) administration. In singly housed rats, LEW rats made more impulsive choices at baseline compared to F344 rats. In addition, impulsive choices decreased for LEW rats but increased or did not change for F344 rats when $d$ AMP was administered. The present study found the reverse in pair-housed rats: F344 rats made more impulsive choices at baseline than LEW rats. In addition, $d$-AMP either increased or did not affect impulsive choices in LEW rats, but decreased impulsive choices in F344 rats. Though a small sample size limits the conclusions that may be drawn from the data, this study demonstrates that environmental variables, particularly social conditions, may affect impulsive choice in the LEW and F344 strains differently. In addition, effects of $d$-AMP on impulsive choice are likely baseline-dependent, evidenced by the occurrence of decreases in impulsive choice in response to $d$-AMP for rats making larger numbers of baseline impulsive choices in both studies. 


\section{ACKNOWLEDGEMENTS}

I would like to thank Dr. Karen Anderson, Dr. Elizabeth Kyonka, and Dr. Melissa Blank for serving on the committee and providing insightful feedback on the project. I would like to express special thanks to my adviser, Dr. Karen Anderson, for her dedicated mentorship, guidance, and support throughout my graduate career. I am especially grateful for Dr. Elizabeth Kyonka's phenomenal teaching and for sharing her invaluable knowledge of statistics with me. Thank you to my big lab-siblings, Chris Krebs and Meagan Follett for their teaching and support. Thank you to Jenny Ozga, Ashley Shultz, and Amanda Press for their help in the lab while conducting this experiment. Thank you (always) to my parents, Frank and Diane Turturici, who have been an unending source of love and support. Finally, I would like to thank my friends at WVU and abroad for their encouragement and laughs: Paul Enlow, Elizabeth Edens, Trisha Hopkins, Rebecca Delaney, Carolyn Rudy, Apral Foreman, and Rachel Stoiko. 


\section{TABLE OF CONTENTS}

Page

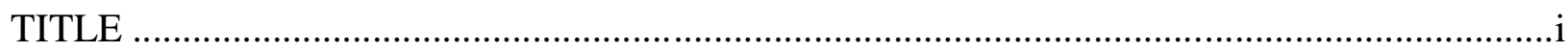

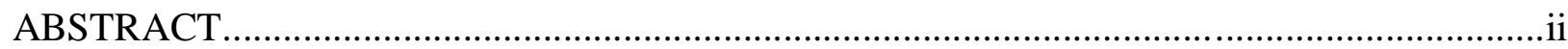

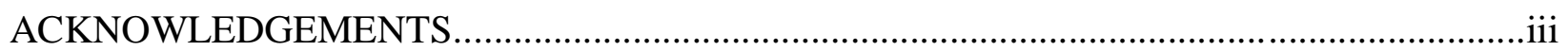

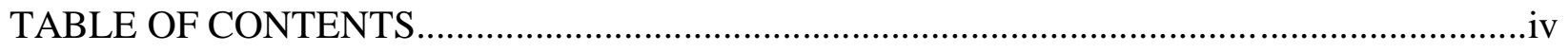

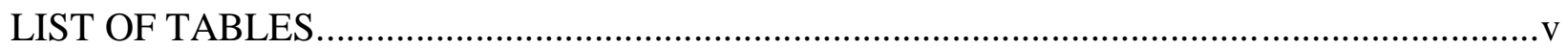

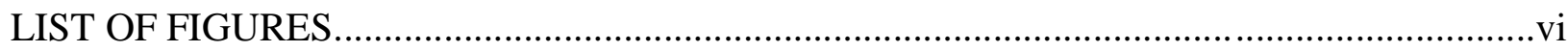

INTRODUCTION

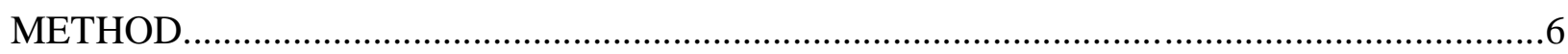

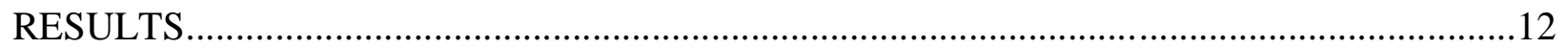

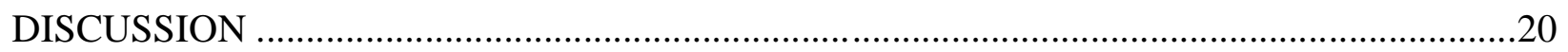

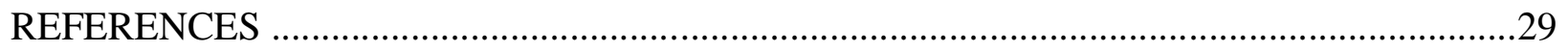

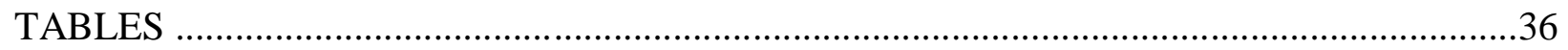

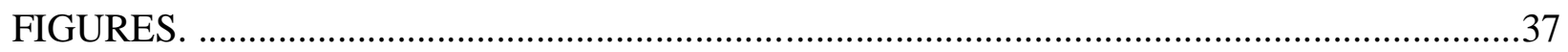




\section{LIST OF TABLES}

Table 1

.36

Indifference points at baseline and area under the curve (AUC) at baseline and at each acute administration of $d$-Amphetamine 


\section{LIST OF FIGURES}

Figure 1

Average percent larger-reinforcer choice as a function of delay for the last 10 sessions of baseline and during the last 0 -s probe sessions before drug administration

Figure 2

Indifference points for paired and singly housed rats from the last 10 sessions of the baseline condition

Figure $3 \mathrm{a}$

Percent larger-reinforcer choice as a function of delay for singly housed rats

Figure $3 b$.

Percent larger-reinforcer choice as a function of delay for pair-housed rats

Figure 4.

Area under the curve as a function of dose for paired and singly housed rats

Figure 5 .

Area under the curve as a function of dose collapsed across singly housed and pair-housed rats 
Effects of Paired Housing and Acute $d$-Amphetamine Administration on Delay Discounting in Lewis and Fischer 344 rats

Impulsive actions underlie many behavioral disorders. For example, impulsivity is correlated with substance abuse (Bickel, Odum, \& Madden, 1999; Kirby, Petry, \& Bickel, 1999; Madden, Petry, Badger, \& Bickel, 1997), obesity (Weller, Cook, Avsar, \& Cox, 2008), attentiondeficit/hyperactivity disorder (ADHD; Schweitzer \& Sulzer-Azaroff, 1995; Sagvolden, Aase, Zeiner, \& Berger 1998, Solanto et al., 2001), pathological gambling (Alessi \& Petry, 2003; Petry, 2001; Reynolds, 2006) and other impulse-control disorders. A common operational definition of impulsivity is the choice of a smaller, more immediate reinforcer, over a larger, delayed reinforcer (Ainslie, 1975; Mazur, 1987; Perry \& Carroll, 2008). Conversely, a selfcontrol choice is defined as choice of a large, delayed reinforcer over a smaller, more immediate reinforcer. Such choices can be studied in delay-discounting procedures, in which more choices for the smaller, more immediate reinforcer indicate increased delay discounting, and greater impulsive choice.

There are several types of procedures used to measure delay discounting. In one procedure using discrete trials, the delay to the delivery of the larger reinforcer is increased across blocks of trials over the course of a session (Evenden \& Ryan, 1996; 1999). At the beginning of the session, when both reinforcers are available immediately, choice is usually exclusive or nearly exclusive for the larger reinforcer. As the delay to the larger reinforcer increases, choice for the smaller, more immediate reinforcer (the impulsive choice) increases systematically. When measuring percent larger-reinforcer choice across a range of delays, a delay-discounting function can be obtained (Anderson \& Woolverton, 2005; Anderson \& Diller, 2010; Evenden \& Ryan, 1996, 1999; Huskinson \& Anderson, 2012; Huskinson, Krebs \& 
Anderson, 2012). Steeper delay-discounting functions indicate more impulsive choices. The delay-discounting function allows quantitative measures of delay discounting to be obtained, including indifference points (Bickel et al., 1999; Mazur, 1987), and area under the delaydiscounting curve (AUC; Myerson, Green \& Warusawitharana, 2001). Indifference points indicate the delay value at which the larger reinforcer constitutes $50 \%$ of total choice (Bickel et al., 1999; Anderson \& Woolverton, 2005; Huskinson et al., 2012). Higher AUC values and longer indifference points indicate less impulsive choice, or more self-control choice (Myerson et al., 2001). Generally, individuals with various impulse-control disorders, such as pathological gambling and substance abuse, have steeper delay-discounting functions, lower AUC, and shorter indifference points than those without behavioral disorders (e.g., Alessi \& Petry, 2003; Bickel, Odum, \& Madden, 1999; Petry, 2001). AUC and indifference points have also been shown to vary across rat strains, particularly Lewis (LEW) and Fischer 344 (F344) rats, and can be altered by drug administration (Anderson \& Woolverton, 2005; Huskinson \& Anderson, 2012; Huskinson, Krebs, \& Anderson, 2012; Wilhelm \& Mitchell, 2009).

\section{Lewis and Fischer 344 Rats}

The LEW and F344 rat strains have been used to study genetic differences in delay discounting in a number of experiments. LEW rats make more impulsive choices (Anderson \& Diller, 2010; Anderson \& Woolverton, 2005; Madden, Smith, Brewer, Pinkston, \& Johnson, 2008; Stein, Pinkston, Brewer, Francisco, \& Madden, 2012) and have shorter indifference points (Anderson \& Diller, 2010; Anderson \& Woolverton 2005; Huskinson et al., 2012) in delaydiscounting tasks than F344 rats when singly housed.

LEW rats may make more impulsive choices than F344 rats due to their differences in neurochemistry. For example, there is a disparity in levels of dopamine and serotonin receptors 
and transporters in several brain areas between the two strains (Burnet, Mefford, Smith, Gold, \& Sternberg, 1992; Flores, Wood, Barbeau, Quirion, \& Srivastava 1998; Selim \& Bradberry 1996). See Huskinson et al. (2012) for an overview of neurochemical differences between LEW and F344 rats. These differences might account for some of the disparity in the number of impulsive choices made between these strains. Research suggests that one or more of these monoamines (i.e., dopamine and serotonin) are involved in impulsive choice (see Winstanley et al., 2006, for a review).

$d$-Amphetamine ( $d$-AMP) induces release of dopamine in brain areas involved in reinforcement processes, and also has some effects on serotonin levels (Kuczenski \& Segal, 1999). Acute $d$-AMP generally decreases delay discounting in various strains of rats (Cardinal, et al. 2000; deWit, Enggasser, \& Richards, 2002; Huskinson et al., 2012; Richards, Sabol, \& deWit, 1999; Wade, deWit, \& Richards, 2000). However, Huskinson et al. (2012) found effects of $d$ AMP depended on baseline rates of delay discounting, which varied among the LEW and F344 strains when singly housed. LEW rats had shorter baseline indifference points (made more impulsive choices), and for these rats, acute $d$-AMP dose dependently decreased rate of delay discounting (lowered number of impulsive choices). However, at the same doses, $d$-AMP increased delay discounting (increased number of impulsive choices) in the F344 rats. The neurochemical differences between LEW and F344 rats may also account for the differential responses to $d$-AMP observed in these strains. It is unknown whether the genetic differences in delay discounting and differential response to $d$-AMP on delay discounting can be attenuated through environmental modifications. For example, it is unknown whether differences in impulsive choice will still be apparent in LEW and F344 rats that are housed in pairs rather than individually. 


\section{Environmental Enrichment and Delay Discounting}

Environmental enrichment is a broad term describing social and/or physical stimulation that supplements the standard housing and care of a laboratory animal (Stewart \& Bayne, 2004). Physical enrichment includes placement of toys or other objects in the animal's home cage, while social enrichment is defined as paired or group housing of animals in the same home cage. Physical and social enrichment are sometimes used simultaneously (Simpson \& Kelly, 2011). Environmental enrichment of both types has been shown to alter neurochemistry in the rat brain (Simpson \& Kelly, 2011; Solinas, Thiriet, Chauvet, \& Jaber, 2010), and thus may alter delay discounting.

Housing conditions have been shown not only to affect delay discounting, but also $d$ AMP's effects on delay discounting. Perry et al. (2008) demonstrated that Sprague-Dawley rats reared in an enriched environment with both toys and conspecifics in the home cage had lower baseline rates of delay discounting than those housed singly. $d$-AMP dose dependently increased rates of delay discounting in rats reared in enrichment, and decreased rates of delay discounting for singly housed rats. This report provides further support that effects of $d$-AMP depend on baseline rates of delay discounting. However, because rats were exposed to both group housing and physical stimulation, it is unknown which of these variables contributed to the differential rates of delay discounting. It is important to note though, that other studies have shown paired housing of rats without physical enrichment to be sufficient to induce differential behavioral effects in the presence of $d$-AMP, although delay discounting was not measured (e.g., Bardo, Klebaur, Valone, \& Deaton, 2001; Zeeb, Wong \& Winstanley, 2013). It is also unknown what effect paired housing will have on delay discounting in the LEW and F344 strains, specifically. It may be the case that baseline rates of delay discounting still vary between LEW and F344 rats 
after paired housing. This does not necessarily indicate that $d$-AMP will have differential effects on delay discounting. Conversely, even if paired housing affects baseline rates of delay discounting in either or both strains, paired housing may not necessarily change effects of $d$ AMP on delay discounting in these strains.

Although group housing and physical enrichment have been shown to decrease delay discounting in Sprague-Dawley rats, it is not known how it affects strains that already differ in rates of delay discounting. In particular, effects of social housing on LEW and F344 rats are unknown. Thus, it is unclear whether paired housing could "override" the genetic differences that underlie the differential rates of delay discounting in these two strains.

The purpose of the present study was to compare effects of paired and single housing on delay discounting in LEW and F344 rats. Paired housing of LEW and F344 rats could differentially affect their differences in delay discounting. Perry et al. (2008) demonstrated that rats housed in environmental enrichment had lower rates of delay discounting at baseline than rats housed singly, but it is unclear whether this effect was due to the physical or social enrichment component. The present experiment examined whether enrichment by paired housing alone affects delay discounting. In addition, while Perry et al. (2008) showed that environmental enrichment alters delay discounting in Sprague-Dawley rats, the present experiment compared effects of paired housing in the genetically and behaviorally different LEW and F344 rats. To achieve this, data from pair-housed LEW and F344 rats were compared to archival data from Huskinson et al. (2012). Effects of $d$-AMP on delay discounting in pair-housed LEW and F344 rats was also compared to archival data from the same study. Thus, in the present study, all data were collected from pair-housed LEW and F344 rats and compared to archival data from Huskinson et al. (2012). 


\section{Method}

The present study systematically replicated the baseline and acute drug administration phases of Huskinson et al. (2012), with the exception that the LEW and F344 rats were housed in same-strain pairs. Other procedural variations are noted below where applicable. The most notable difference in the procedure was fewer 0-s probe sessions, which tested for discrimination of reinforcer amounts, for pair-housed rats than singly housed rats. Differences in delay discounting between the pair-housed LEW and F344 rats were compared with those of the singly housed LEW and F344 rats from Huskinson et al. (2012).

\section{Subjects}

Six experimentally naïve LEW and six experimentally naïve F344 rats served as pairhoused subjects. Eight experimentally naïve rats of each strain served as singly housed subjects. Rats were obtained from Harlan Sprague-Dawley, Inc. (Indianapolis, IN).

Pair-housed rats were approximately 21 days old when shipped. Rats were housed in same-strain pairs for eight weeks prior to the start of experimentation, because this was sufficient to produce differential rates of delay discounting in Perry et al. (2008), and is a common length of differential rearing in many studies using environmental enrichment ( see Simpson \& Kelly, 2011 for a review). In addition, eight weeks was chosen to ensure that the age of both singly and pair-housed rats was approximately equivalent at the beginning of testing. Thus, all rats were approximately 11 weeks old at the start of testing.

During the eight weeks prior to testing, pair-housed rats were fed standard rat chow ad libitum and allowed continuous access to water. Food restriction began soon after, in which rats were fed $24 \mathrm{~g}$ of standard rat chow per cage one half-hour after the experimental session, equating to $22 \mathrm{~h}$ of food restriction. These rats were pair-housed throughout the duration of the 
study. Singly housed rats were housed in identical conditions prior to testing, received a similar amount of food (12-16 g per rat), and also received $22 \mathrm{~h}$ of food restriction prior to sessions.

All rats were housed in controlled environmental conditions (temperature, 20 degrees C; 12-hour reversed light-dark cycle) and had continuous access to water. Sessions were conducted during the dark phase of the light-dark cycle at the same time each day. Throughout the entire experiment, rats were weighed once per week. All procedures were approved by West Virginia University's Animal Care and Use Committee. Body weight of rats at the start of testing are reported in the results section.

\section{Apparatus}

Experimental sessions were conducted in eight standard operant-conditioning chambers for rats, each enclosed in a melamine sound-attenuating cubicle (Med Associates, VT). Each chamber contained a working area of $30.5 \mathrm{~cm}$ by $24.5 \mathrm{~cm}$ by $21.0 \mathrm{~cm}$, a grid floor, and a $45-\mathrm{mg}$ pellet dispenser with a pellet receptacle that is centered between two retractable response levers. The levers were $11.5 \mathrm{~cm}$ apart from each other and required a force of $0.25 \mathrm{~N}$ for a response to be recorded. The levers are $4.8 \mathrm{~cm}$ wide, elevated $8 \mathrm{~cm}$ from the grid floor, and protruded $1.9 \mathrm{~cm}$ into the chamber. Two $28-\mathrm{V}$ stimulus lights, $2.5 \mathrm{~cm}$ in diameter, are located $7 \mathrm{~cm}$ above each lever. Each chamber contained a $28-\mathrm{V}$ houselight on the wall opposite the wall containing the operanda. A ventilation fan circulated air and masked extraneous noise. Data collection and programmed consequences were controlled by a personal computer equipped with Med-PC software (Med Associates, VT). The operant-conditioning chambers used in the present study were the same chambers used in Huskinson et al. (2012).

\section{Procedure}


Baseline. The experiment began with lever-press training. Both levers were extended into the chamber, and food was delivered according to a tandem fixed-ratio (FR) 1, differentialreinforcement-of-other-behavior 10-s schedule. Sessions ended after $8 \mathrm{~h}$. If lever pressing was not established after this session, it was shaped using reinforcement of successive approximations. After lever-press acquisition, rats were trained on an alternating FR 1 schedule of reinforcement. Both levers were extended into the chamber and a cue light above one randomly determined lever was illuminated. Each response on that lever resulted in delivery of one food pellet, while responses on the other lever were counted but had no other programmed consequences. After five food pellets were earned for presses on the designated lever, the position of the illuminated cue light and the FR 1 contingency alternated to the other lever. The contingency alternated for presses on each lever until 40 food pellets were delivered. Sessions continued until food pellets were reliably earned for presses on both levers.

The delay-discounting procedure used was identical to that of Huskinson et al. (2012), from which data comparisons were made. Each session began with a 10-min blackout. Sessions consisted of five blocks of eight trials each, which began every $100 \mathrm{~s}$. This resulted in inter-trial intervals (ITIs) of varying durations. The first two trials of each block were forced-exposure trials, in which the houselight was illuminated and one randomly determined lever was extended into the chamber with its respective cue light lit. If a response occurred on this lever, it retracted, its respective cue-light darkened, and either one food pellet immediately or three food pellets after a delay were delivered. On the second forced-exposure trial, the other lever was extended with its respective cue light illuminated. If a response occurred on that lever, it retracted, its respective cue light darkened, and the other consequence occurred (i.e., one pellet immediately or three food pellets after a delay). One of each forced-exposure trial occurred and the next six 
trials were free-choice trials. During a free-choice trial, both levers extended, each with their respective cue lights illuminated, and choice was between the two alternatives. If a response was made on either lever, the consequence associated with that lever followed. When a small reinforcer was delivered, the houselight flashed as the food pellet was dispensed into the food trough, and then remained off for the remainder of the trial. When a large reinforcer was delivered, the houselight remained on during the delay, flashed three times when the three food pellets were dispensed into the food trough, and remained off for the remainder of the trial. If a lever press was not made within $30 \mathrm{~s}$ of a trial onset, that trial was recorded as an omission, the lever(s) retracted, cue light(s) and houselight darkened, and a 70-s ITI began. If six or more omissions occurred on free-choice trials, the data from that session were excluded from analyses. Originally, the lever paired with the larger reinforcer for each rat was counterbalanced across LEW and F344 strains, such that half of the LEW rats and half of the F344 rats were trained with the larger reinforcer available for right lever presses, and the remaining half of each group was trained with the larger reinforcer available for left lever presses. Originally, three rats of each strain were randomly assigned to respond on the left lever for the larger reinforcer and the remaining three rats of each strain were assigned to respond on the right lever for the larger reinforcer. Due to lever biases (exclusive choice for one of the levers and/or several omissions of forced-exposure trials on the other lever) in three rats, three rats reached stability with the larger reinforcer on the left lever and nine rats reached stability with the larger reinforcer on the right lever.

Training began with both consequences delivered immediately to establish discrimination of reinforcer amounts. When choice for the larger reinforcer reached at least $80 \%$, delays to the larger reinforcer were increased across blocks. The delay to the smaller reinforcer was always set 
at $0 \mathrm{~s}$. The first delay series presented increasing delays of $0,2,4,8$, and $16 \mathrm{~s}$ across blocks. In this delay series, in the first block, the six free-choice trials had a larger-reinforcer delay of $0 \mathrm{~s}$. In the second block, the six free-choice trials had a larger-reinforcer delay of $2 \mathrm{~s}$. In the third block, the six free-choice trials had a larger-reinforcer delay of $4 \mathrm{~s}$. This procedure continued until the five blocks were completed. The delay series was adjusted as necessary to obtain delaydiscounting functions without floor (near exclusive choice for the smaller reinforcer) or ceiling (near exclusive choice for the larger reinforcer) effects. The longer delay series included delays of $0,5,10,20$, and $40 \mathrm{~s}$, or $0,10,20,40$, and $60 \mathrm{~s}$. When no floor or ceiling effects were apparent, that delay series was considered the terminal delay series, which varied between rats. Sessions ended after 40 total trials and consisted of a total of 10 forced-exposure and 30 freechoice trials. Sessions were conducted five days per week.

Following determination of a terminal delay series for a rat, a baseline delay-discounting function was established. Baseline sessions were conducted for a minimum of ten sessions and until responding reached stability. The criteria for stability were defined as no increasing or decreasing trends in total larger-reinforcer choice in free-choice trials across the last five sessions, less than $20 \%$ variation in the total larger-reinforcer choice during each of the last five sessions from the grand mean, and at least $80 \%$ or greater larger-reinforcer choice in the 0 -s delay block. To test for discrimination of reinforcer magnitude and delay, when responding reached stability, a 0 -s probe session was conducted during which all delay values were set equal to zero (Cardinal et al., 2000; Diller, Saunders, \& Anderson, 2008; Evenden \& Ryan, 1996, 1999; Huskinson et al. 2012). Subsequent probe sessions were conducted if larger-reinforcer choice was less than $80 \%$ in one or more blocks. $d$-AMP administration began when stable responding was reestablished on the terminal delay series. The procedure of Huskinson et al. 
(2012) included 0-s probe sessions each Wednesday. Due to time constraints that were not present in Huskinson et al. (2012), fewer 0-s probe sessions were conducted in the present study.

Acute $\boldsymbol{d}$-AMP administration. The same delay-discounting procedure used to establish baseline data was used to assess acute effects of $d$-AMP. Saline or $d$-AMP $(0.1,0.3,1.0,1.7$ $\mathrm{mg} / \mathrm{kg}$ ) was administered to all rats. Drug administration occurred on every Tuesday and Friday if larger-reinforcer choice was at least $80 \%$ in all blocks during the last 0 -s probe session and was at least $80 \%$ in the first free-choice block (when both delay values are $0 \mathrm{~s}$ ) during the session immediately prior to drug administration (control sessions). Thus, control sessions occurred each Monday and Thursday, while Wednesday's sessions were identical to control sessions. Sessions on Tuesday and Friday were also identical to control sessions if the criteria for saline or $d$-AMP administration was not met. Effects of saline were determined for at least two sessions prior to $d$ AMP administration to allow habituation to the injection procedure. Doses of $d$-AMP were administered in both an ascending and descending sequence, with an additional saline administration occurring between these sequences. Each dose of $d$-AMP was tested at least twice. Additional doses followed if high variability in larger-reinforcer choice was observed during drug-administration days.

\section{Data Analysis}

Percent larger-reinforcer choice was the primary dependent variable. Delay-discounting functions were plotted for each rat as percent larger-reinforcer choice across each delay amount in the terminal delay series. Indifference points and AUC were calculated using the same method as Huskinson et al. (2012), by using nonlinear regression. AUC was then calculated by drawing vertical lines from the normalized $\mathrm{x}$-axis to each obtained percent larger-reinforcer choice at each delay. The area of the trapezoids formed by this manipulation was calculated and divided 
by the whole area of the graph. The indifference point was determined by interpolating the delay value at which larger-reinforcer choice is 50\% (Myerson et al., 2001). Indifference points and AUC were compared to archival data from the singly housed rats of Huskinson et al. (2012). Two $t$-tests were conducted on AUC, with each testing for differences in AUC between LEW and F344 - one test for pair-housed rats and one test for singly housed rats. AUC tends to be similar across rats when delay-discounting functions are functionally equivalent (without any floor or ceiling effects), even when rats respond on different delay series. Indifference points vary between rats because the measure depends on the number of seconds in the terminal delay value. Number of sessions required to reach stability and number of 0 -s probe sessions were also calculated and compared across rat strain and housing type using $t$-tests. A 2 × 2 mixed-factorial ANOVA was conducted to evaluate effects of housing type and rat strain on indifference points at baseline. Rat strain and housing condition served as between-subjects variables, while mean AUC and indifference points served as the within-subjects variables.

Indifference points and AUC were compared to archival data obtained after $d$-AMP administration by Huskinson et al. (2012). Indifference points and AUC were also compared to data obtained from Experiment 1. A $2 \times 2 \times 4$ mixed-factorial ANOVA test was conducted to evaluate effects of housing type, rat strain, and $d$-AMP dose on AUC and indifference points. Rat strain and housing condition served as between-subjects variables, while $d$-AMP dose served as the within-subjects variable. Responding in the control block was also analyzed using a 2 x 2 × 3 ANOVA with the same between-subjects variables, and session type (control, saline, or drug) as the within-subjects variable.

\section{Results}

\section{Body Weight}


Singly housed LEW rats were heavier than singly housed F344 rats, but the opposite effect occurred for pair-housed rats throughout the duration of the study. Singly housed LEW rats weighed an average of $335 \mathrm{~g}(\mathrm{SEM}=5.7)$ and singly housed F344 rats weighed an average of $280 \mathrm{~g}(\mathrm{SEM}=4.7)$ at the beginning of testing. Pair-housed F344 rats weighed an average of $268 \mathrm{~g}(\mathrm{SEM}=4.4)$ and pair-housed LEW rats weighed an average of $287 \mathrm{~g}(\mathrm{SEM}=9.0)$. The difference in weights between the strains at the beginning of testing was significant for singly housed rats, $t(7)=58.86, \mathrm{p}<.01$, but not pair-housed rats. Weights of pair-housed rats were averaged throughout the study and given a rank of " 1 " or " 2 " based on whether the rat had a higher or lower average weight throughout the study than its cage mate. Though not significant, for pair-housed LEW rats, having a higher weight than the cage mate was positively correlated with indifference points, $r_{s}(4)=0.29$, while for pair-housed F344 rats a negative correlation was found $r_{s}(4)=-0.29$. For some pairs, the difference between body weight of one rat relative to its cage mate was consistently over $10 \mathrm{~g}$, while for other pairs body weight was more similar throughout the study. Statistics were not conducted on differences in body weight between cage mates because this effect was not strain-specific. In addition, the sample size was too small to detect an effect (3 pairs per strain).

\section{Baseline}

All sessions prior to the first injection of the experiment were considered part of the baseline phase. Three pair-housed rats showed lever biases (exclusive choice for the larger reinforcer) during the baseline phase. The lever associated with the larger reinforcer was reversed for these rats. Two of the three pair-housed rats showing lever biases were LEW rats, and all were responding on the left lever. Thus, for 9 of 12 rats, the right lever was associated with the larger reinforcer at the end of the study. 
Like the singly housed rats, pair-housed LEW rats took longer to reach stability than pairhoused F344 rats. In Huskinson et al. (2012), singly housed LEW rats required a mean of 94.6 sessions $(\mathrm{SEM}=15.8)$ to reach stability, and singly housed F344 rats required a mean of 60.5 sessions $(\mathrm{SEM}=8.0)$ to reach stability. In the present study, pair-housed LEW rats required a mean of 83.83 sessions $(\mathrm{SEM}=18.52)$ and pair-housed $\mathrm{F} 344$ rats required a mean of 45.17 sessions $(\mathrm{SEM}=5.47)$ to reach stability. The differences in sessions to reach stability were not significant for paired or singly housed rats.

A decrease in percent larger-reinforcer choice was observed with increases in delay to the larger reinforcer for both pair-housed and singly housed rats. Figure 1 shows percent largerreinforcer choice from the last 10 baseline sessions of the experiment (circles). Data from 0-s probe sessions are also presented in Figure 1 (squares). Panels are divided based on rat strain and delay series. Closed symbols indicate data from singly housed rats while open symbols indicate data from pair-housed rats. Data from 0-s probe sessions demonstrate choice was at or above $80 \%$ when the delay to the larger reinforcer was 0 s. One exception occurred for the pair-housed LEW rat responding in the 16-s delay series (top left panel). Because the percent largerreinforcer choice is averaged across 0 -s probe sessions, it is not the case that this rat did not reach $80 \%$ across all blocks of trials but that it required several 0 -s probe sessions to do so. A direct comparison between paired and singly housed rats cannot be made across 0 -s probe sessions because in the present study 0-s probes were much less frequent than in Huskinson et al. (2010). However, choice shifted similarly in both pair-housed and singly housed rats when 0-s probes were introduced, evidenced by the contrast between the baseline and 0 -s probe functions in Figure 1. Baseline delay-discounting functions appear similar across all strains, housing types, and delay series, demonstrating that delay series were functionally equivalent across groups. A 
slight difference may lie in the data for the LEW rats in the 60-s delay series (all pair-housed, top right panel), which display a slightly flatter delay discounting function. However, a difference in this function was not supported by statistical analyses of AUC values.

Functionally equivalent delay series weredemonstrated via baseline AUC values. Pairhoused F344 rats had a mean AUC of $0.43(\mathrm{SEM}=0.03)$ at baseline, while pair-housed LEW rats had a mean AUC of $0.45(\mathrm{SEM}=0.07)$. Singly housed LEW rats had a mean AUC of 0.43 (SEM $=0.03)$ and singly housed F344 rats had a mean AUC of $0.46(\mathrm{SEM}=0.04)$. Table 1 shows individual-subject AUC values at baseline and following administration of each dose of $d$-AMP for all rats. There were no significant effects of strain or housing type on AUC at baseline.

As presented in Figure 1, choice stabilized on different delay series for paired and singly housed LEW and F344 rats. For example, in the singly housed rats, most LEW rats responded on the 16-s delay series, while only one pair-housed LEW rat responded in this delay series. Similarly, no singly housed F344 rats responded in the 16-s delay series, in contrast to 4/6 pairhoused rats. In addition, no singly housed LEW rats responded in the 60-s delay series, in contrast to $3 / 6$ pair-housed rats. Because paired and singly housed rats of each strain did not respond in each delay series, terminal delay series was not used as a factor in statistical analyses, though it was in Huskinson et al. (2012).

The delay series in which stable responding is established also somewhat reflects the value of the indifference point. For example, with similarly shaped delay-discounting functions, choice in the 16-s delay series will yield a shorter indifference point than choice in the 40-s delay series. Thus, pair-housed LEW and F344 rats displayed indifference points that were in the opposite direction of singly housed LEW and F344 rats. Figure 2 shows that singly housed F344 rats had longer indifference points than singly housed LEW rats, yet pair-housed F344 rats had 
shorter indifference points than pair-housed LEW rats. Table 1 and Figure 2 show the mean indifference points for all rats from the last ten sessions of the baseline condition. Singly housed F344 rats had the longest mean indifference point (mean $=20.54, \mathrm{SEM}=3.67)$, followed by pair-housed LEW rats $($ mean $=18.75, \mathrm{SEM}=7.60)$, singly housed LEW rats $($ mean $=10.04$, $\mathrm{SEM}=2.41)$, and finally, pair-housed F344 rats $($ mean $=6.10, \mathrm{SEM}=1.04) . \mathrm{A} 2 \mathrm{x} 2$ factorial ANOVA revealed no significant main effect of strain or housing type, but did reveal a significant interaction between these variables on indifference points, $F(1,24)=7.77, \mathrm{p}=.01$. Pair-wise comparisons using Fisher's Least Significant Difference indicated a significant difference between singly housed F344 rats and pair-housed F344 rats $(\mathrm{p}<.05)$. These tests indicate that F344 rats' baseline indifference points were shorter when pair-housed than when singly housed. Though LEW rats appeared to have longer indifference points when pair-housed than singly housed, this effect was not significant. Pair-housed LEW rats displayed more variability in their indifference points than pair-housed F344 rats. For example, indifference points in pair-housed LEW rats ranged from 2.7 to $54.6 \mathrm{~s}$, while indifference points in pair-housed F344 rats ranged from 2.8 to 10.4 s.,

\section{Acute $d$-AMP Administration}

Percent larger-reinforcer choice as a function of delay for each dose of $d$-AMP is shown in Figure 3a (singly housed rats) and 3b (pair-housed rats). Data presented in each panel are grouped by housing condition, strain, and delay series. One of the pair-housed LEW and three of the pair-housed F344 rats made more than six response omissions following administration of $1.7 \mathrm{mg} / \mathrm{kg} d$-AMP. Data from this dose were not included in the statistical analyses, though data from sessions in which fewer than six response omissions occurred are still presented in Figures 3 (open squares), and 5. As in Huskinson et al. (2012), indifference points could not be 
interpolated for several determinations of each dose of $d$-AMP due to percent larger-reinforcer choice increasing above 50\%. Thus, AUC was used as the main dependent variable after $d$-AMP administration began.

For the singly housed rats presented in Figure 3a, $d$-AMP dose dependently increased larger-reinforcer choices for LEW rats only (left panels). Filled symbols indicate data from saline administration and open symbols indicate doses of $d$-AMP. For the singly housed F344 rats, $d$ AMP either decreased or did not affect percent larger-reinforcer choice, and high doses of $d$ AMP lowered choice in the first block of trials (when the delay to the larger-reinforcer is $0 \mathrm{~s}$ ) below $80 \%$. In Figure 3a, when the delay was 0 -s, open symbols in the right panels lie below $80 \%$ for many doses of $d$-AMP demonstrating the disruption of responding in the control block.

For the pair-housed rats presented in Figure 3b, $d$-AMP dose dependently increased percent larger-reinforcer choice in F344 rats (right panels), but had unclear effects or decreased percent larger-reinforcer choice in LEW rats (left panels). Thus, effects of rat strain were in the opposite direction of those from Huskinson et al. (2012). There was one exception to this effect, rat L5P. This rat was also the only pair-housed LEW rat responding in the 16-s delay series. For this rat (top left panel), percent larger-reinforcer choice increased when $d$-AMP was administered, similar to that of all rats responding in the 16-s delay series. This is also shown in Table 1. Thus, choice at baseline and under $d$-AMP was similar across all rats responding in the 16-s delay series, regardless of strain or housing condition (Figure 3a: top left panel, Figure 3b: top two panels). The decreases in percent larger-reinforcer choice in the control block mentioned above can be seen at the dose of 1.0 (diamonds) in nearly all pair-housed rats (Figure 3b) and singly housed F344 rats responding in the 60-s delay series (Figure 3a, bottom right panel). The 
dose of 1.7 (squares) also had a decreasing effect on responding in the control block in many cases.

Figure 4 shows mean AUC at baseline and following each dose of $d$-AMP for both pairhoused and singly housed rats. A similar effect of delay series can be seen in the data from the 16-s delay series (two top panels). Like percent larger-reinforcer choice, AUC increased regardless of strain in this delay series. Choice in the 40- and 60-s delay series appeared to be more strain-dependent (middle panels). For singly housed rats, AUC increased for LEW rats and decreased for F344 rats. For pair-housed rats, AUC decreased for LEW rats while F344 rats showed individual variability (AUC increased for one rat and decreased for the other). Data from the rats responding in the 60-s delay series showed dose-dependent decreases in singly housed rats (F344 rats only, bottom left panel of Figure 4). AUC from pair-housed rats responding in the 60-s delay series increased slightly, particularly at the $1.7 \mathrm{mg} / \mathrm{kg}$ dose (bottom right panel).

The $2 \times 2 \times 4$ mixed factorial ANOVA revealed a significant within-subjects main effect of dose $F(1.62,38.75)=7.99, \mathrm{p}<.01$, and no significant interactions. The mixed-factorial ANOVA revealed no significant between-subjects effects or interactions. Thus, the data were collapsed across rats. Average AUC for each dose of $d$-AMP for all rats is shown in Figure 5. $d$ AMP had an increasing effect on AUC, on average. In the mixed factorial ANOVA, Mauchly's Test of Sphericity was significant, $\chi^{2}(5)=32.01, \mathrm{p}<.001$, so the Greenhouse-Geisser corrected degrees of freedom were used. Pair-wise comparisons using the Bonferroni test revealed significant differences between saline and $0.3 \mathrm{mg} / \mathrm{kg}$, and between 0.1 and $0.3 \mathrm{mg} / \mathrm{kg}$.

In sum, singly housed LEW rats showed increases in AUC at all doses of $d$-AMP, while singly housed F344 rats showed increases in AUC generally only at lower doses of $d$-AMP (0.1 and $0.3 \mathrm{mg} / \mathrm{kg}$ ). The pair-housed LEW rat responding in the 16-s delay series showed increases 
at all doses of $d$-AMP, but pair-housed LEW rats in the 40- and 60-s delay series displayed mixed results. Pair-housed F344 rats generally showed increases at all doses of $d$-AMP, but again this result may be delay-series dependent.

$d$-AMP disrupted responding in the control (0-s) block for pair-housed LEW rats more than pair-housed F344 rats. In pair-housed rats, the decline below 80\% in control-block largerreinforcer choice can be seen in LEW rats at doses of 1.0 and 1.7, and F344 rats at doses of 1.0 (Figure 3b). This effect was also in the opposite direction of singly housed rats (Figure 3a), in which $d$-AMP disrupted responding in the control block for F344 rats to a greater extent. Only in pair-housed LEW rats was responding in the control block for $d$-AMP sessions below $80 \%$, on average (69.12, SEM = 3.4, Figure 3b: left panels). Responding in the control block of trials under $d$-AMP depended on both strain and housing type. For F344 rats, responding in the control block of trials appears to decrease under several doses of $d$-AMP regardless of housing type, while LEW rats' responding in the control block was mainly affected when pair-housed. A 2 x 2 x 3 mixed-factorial ANOVA was conducted on control block responding across session type (Control, Saline, or Drug days) and revealed a significant main effect of session type on percent larger-reinforcer choice in the control block within rats, $F(1.28,30.59)=44.48$, p <.001. Pairwise comparisons using the Bonferroni test revealed a significant difference between $d$-AMP sessions and control sessions, and between $d$-AMP sessions and saline sessions (both $\mathrm{p}<.001$ ). The between-subjects tests revealed a main effect of strain, $F(1,24)=11.56, \mathrm{p}<.01$, a main effect of housing type, $F(1,24)=17.79, \mathrm{p}<.001$, and a significant strain $\mathrm{x}$ housing type interaction, $F(1,24)=9.01, \mathrm{p}<.01$, on percent larger-reinforcer choice in the control block. The within-subjects effects also showed a significant session type $\mathrm{x}$ strain $\mathrm{x}$ housing type interaction, $F(1.28,30.59)=7.01, \mathrm{p}<.01$. Mauchly's Test of Sphericity was significant, $\chi^{2}(2)=19.36, \mathrm{p}<$ 
.001 , so the Greenhouse-Geisser corrected degrees of freedom were used. The interaction indicates that responding in the control block was disrupted during $d$-AMP sessions most for F344 rats when singly housed, but disrupted most for LEW rats when pair-housed. In addition, responding in the control block for LEW rats was lower than F344 rats during control and saline sessions regardless of housing conditions.

\section{Discussion}

For the singly housed rats of Huskinson et al. (2012), LEW rats had shorter indifference points than F344 rats at baseline. When $d$-AMP was administered, AUC increased for LEW rats and decreased or remained the same for F344 rats. Paired housing appears to have different effects on LEW and F344 rats. Pair-housed LEW rats displayed longer indifference points and pair-housed F344 rats displayed shorter indifference points, as compared to their singly housed counterparts. This effect was only significant for F344 rats. The large amount of variability in indifference points for LEW rats and the small sample size may have prevented detection of a significant difference. $d$-AMP increased AUC in pair-housed rats but individual differences were observed across rats of both strains. Effects of $d$-AMP also appeared to be baseline dependent.

Garcia and Kirkpatrick (2013) found that pair-housed LEW rats were more impulsive than pair-housed Wistar rats. (LEW rats were bred from Wistar rats and thus Wistar rats are often used as their control.) Garcia and Kirkpatrick (2013) used a different delay-discounting task in which response-initiated fixed-interval schedules of varying durations were interspersed with non-reinforced probe trials. The differences between Wistar and LEW rats were only significant during certain procedural manipulations. For example, when the delay to a smallersooner reinforcer was $15 \mathrm{~s}$ and the delay to a larger-later reinforcer was $30 \mathrm{~s}$, choice for the smaller-sooner reward was significantly greater in LEW than Wistar rats. Other procedural 
differences from the present study include the magnitude of reward, which varied throughout the study ( 1 pellet vs. 2 pellets in the above example). In addition, F344 rats were not compared to LEW rats. Thus, the results of Garcia and Kirkpatrick (2013) cannot be directly compared to the present study, but may point to the importance of procedural variables and strains compared in the examination of impulsive choice. It is unclear why pair-housed LEW rats' choices were impulsive compared to their comparison strain in the above study, but were more self-controlled compared to their comparison strain in the present study.

The delay-discounting functions for pair-housed LEW rats responding in the 60-s delay series appear unusually flat compared to the other groups. Response perseveration may have played a role in baseline rates of impulsive choice in pair-housed rats. Two of three rats that showed persistent lever biases were LEW rats. It is possible that pair-housed LEW rats are more susceptible to response perseveration than pair-housed F344 rats, though when the lever paired with the larger reinforcer was reversed these biases disappeared. Madden et al. (2008) used a procedure that corrected for response biases and still found that singly housed LEW rats made more impulsive choices than singly housed F344 rats. In addition, Stein et al. (2012) did not find that forced-choice trials increased the likelihood of perseveration on the forced-choice lever. Thus, it seems unlikely that the differences seen in pair-housed LEW and F344 rats are due to response perseveration.

The body weights of the LEW and F344 rats used in the present study did not correspond to the typical growth pattern of these strains (Harlan Laboratories, Inc., 2014 a; 2014b). LEW rats weighed significantly less than F344 rats, the opposite effect of Huskinson et al. (2012), and of Huskinson and Anderson (2012). Although the cages used in the present study were the same size, it is possible that the presence of a conspecific in the home cage induced different behavior 
in F344 and LEW rats. For example, F344 rats may have competed for food to a greater extent than LEW rats. If that is the case, their shorter indifference points may point to a subjective difference in food deprivation between the strains. Studies have found mixed results on the effect of food deprivation level on delay discounting, and results are thought to depend on procedural variables such as delay value (Ho, Wogar, Bradshaw, \& Szabadi, 1997). Even though food intake did not differ between the two pair-housed strains, food intake may have differed between each individual rat in a pair. As there were only three pairs, there was insufficient power to detect a strain-specific effect. All rat pairs were fed $24 \mathrm{~g}$ of rat chow per day and all food was eaten prior to each experimental session. However, for some pairs, one rat was consistently a higher weight than the other rat throughout the study. Other pairs were more similar to each other, but again, a strain-specific effect could not be found. Though nonsignificant, for LEW rats, there was a positive correlation between having a higher body weight throughout the study and indifference points, while a negative correlation was found for F344 rats. Future research might use a larger sample of pair-housed LEW and F344 rats to determine if differences in food intake between cage mates affects delay discounting in a strain-specific way.

There is evidence to suggest that LEW and F344 rats behave differently in social situations. Berton, Ramos, Chaouloff, \& Mormede (1997) found that LEW rats engaged in significantly more interactions in an aversive environment (an open field) than F344 rats even though they engaged in a similar amount of locomotor activity. In a neutral environment, LEW and F344 rats engaged in similar amounts of social interaction overall, but differed significantly in specific types of interactions. When they did differ significantly, LEW rats engaged in more of these interactions than F344 rats (e.g., biting attacks, fighting, and being on top of the other rat). If pair-housed LEW rats engaged in more social interactions than pair-housed F344 rats, this 
difference may have affected food intake, which may have affected impulsive choice. Another possibility is that F344 rats' tendency not to engage in social interactions prevented full implementation of the independent variable (paired housing, i.e., enrichment), resulting in shorter indifference points for F344 rats.

It is unclear if these social interactions affect one rat in a pair differently than the other. For example, it is unclear if dominant rats are more likely to have shorter indifference points. However, the positive correlation between having a higher weight and indifference points suggests that this could be true for LEW rats - which presumably engage in more dominancerelated behaviors. Examination of social dominance requires measurement of other variables besides body weight, however. It would be interesting to determine if the same differences in impulsive choice exist between pair-housed LEW and F344 rats when they are fed separately rather than when food is introduced with both rats are present. Perry et al. (2008) fed rats in the enriched condition in separate cages. This was not done in the present study because separating the pairs of rats would have decreased time spent in the enriched environment and could have acted as a stressor.

Perry et al. (2008) provided objects in the home cage of the animals in the enriched condition. Objects were not included in the present study in order to determine if social housing alone has an effect on impulsive choice. Objects may be particularly important in mediating response to $d$-AMP. Hoffmann, Schütte, Koch, \& Schwabe (2009) housed Wistar rats in groups (non-enriched), in groups plus a few objects (low enrichment), and in groups plus many more objects (high enrichment). Acoustic startle response was measured by a motion sensor as the amount of motor movement following presentation of white noise. When vehicle was administered, all groups were similar in acoustic startle response. When apomorphine (a 
dopamine agonist that increases dopamine) was administered, non-enriched (social housing only) groups exhibited the lowest acoustic startle response, followed by the low-enriched group, and then the high-enriched group. For the non-enriched group, acoustic startle response was lower compared to vehicle administration, while for the other two groups acoustic startle response was higher. Although this study did not measure impulsive choice and did not use LEW and F344 rats, it demonstrates that objects plus social housing may affect response to dopaminergic drugs in a way that social housing alone does not. It is important to note that all groups of rats in Hoffman et al. (2009) were fed while together in the home cage.

Perry et al. (2008) demonstrated baseline-dependent effects of $d$-AMP on impulsive choice. Baseline-dependent effects of $d$-AMP were also observed in Huskinson et al. (2012) and in the present study. In the two studies presented here, both singly housed and pair-housed rats responding in the 16-s delay series (the rats showing the lowest indifference points, indicating greatest impulsive choice) showed the largest increases in AUC when $d$-AMP was administered. In Perry et al. (2008), singly housed Sprague-Dawley rats showed higher levels of delay discounting that decreased with $d$-AMP, and group-housed rats of the same strain showed lower levels of delay discounting that increased with $d$-AMP. Perry et al. (2008) used an adjustingdelay procedure, which differs from that of the present study. Craig, Maxfield, Stein, Renda, \& Madden (2014) noted no differences in delay discounting when these two procedures were compared within-subject in Long Evans rats, though LEW and F344 rats may be differentially sensitive to the way delays are presented. For example, in an adjusting-delay procedure, the delay to the larger reinforcer is titrated depending on responses rather than presented in a consistent order across sessions. 
The baseline-dependent effects of $d$-AMP in the present study (and possibly in Perry et al., 2008) may be explained by the modulation of dopamine. $d$-AMP increases dopamine levels. The endogenous action of dopamine may act in an inverted-U-shaped manner, such that intermediate levels of dopamine produce one effect, while both high and low levels of dopamine produce another, but similar effect. For example, Granon et al. (2000) found baseline-dependent effects of dopamine-receptor agonist SKF 38393 (which increases levels of dopamine) on accuracy in a behavioral test designed to measure divided attention and motor impulsivity (the five-choice serial reaction time task). The same dose administered directly to the prefrontal cortex that increased accuracy for initially low-scoring subjects (which presumably began the experiment with lower dopamine levels) decreased accuracy in initially high-scoring subjects (which presumably began the experiment with higher dopamine levels). There is also evidence for an inverted-U-shaped function of dopamine involvement in working memory (Vijayraghavan, Wang, Birnbaum, Williams, \& Arnsten, 2007), such that intermediate levels of dopamine promote working memory function, while low and high levels are detrimental to it.

The action of dopamine in an inverted-U-shaped way might explain the results obtained in the present study. In the case of impulsive choice, both low and high levels of dopamine may result in impulsive choice, whereas intermediate levels of dopamine result in self-control choice. That is, low and high levels of dopamine may result in dysfunction of the dopamine system. Thus, intermediate levels of dopamine might be optimal where self-control choice is concerned. For LEW rats, which have shorter indifference points when singly housed, paired housing may have increased initially low dopamine levels such that self-control choices were increased at baseline to a peak. Then, when $d$-AMP was administered, dopamine continued to increase, resulting in a decrease in self-controlled choices. For F344 rats, which have longer indifference 
points when singly housed, paired housing may have increased already high dopamine levels and resulted in decreased self-controlled choices. The subsequent increase in self-control choice by $d$-AMP in pair-housed F344 rats cannot be explained by the inverted-U-shaped dopamine action, though. Future research might clarify the mechanism by which paired housing alters impulsive choice.

The results of the present study were similar to Huskinson et al. (2012) in that the rats responding in the 16-s delay series showed the greatest increases in percent larger reinforcer choice when $d$-AMP was administered. Unlike Huskinson et al. (2012) however, these rats were predominantly F344 rats (4/5). In Huskinson et al. (2012), all of the four rats responding in the 16-s delay series were LEW rats. Similarly, all F344 rats from Huskinson et al. (2012) responded in either the 40- or 60-s delay series. In the present study, all but one LEW rat (L-5-P) responded in either the 40- or 60-s delay series. Thus, the delay series and strain variables are somewhat confounded. Delay series was not included as a factor in the ANOVA for the present study, though Huskinson et al. (2012) did include it. The reason for this was because no pair-housed F344 rat responded in the 60-s delay series, resulting in empty cells.

It was necessary to change the terminal delay series for each rat to avoid floor and ceiling effects. The advantage of changing the delay series for each rat is that similar AUC values are obtained at baseline, providing equivalent delay-discounting functions. The disadvantage of changing the delay series in the present study was that only one LEW rat (L5P) and two F344 rats (F1P and F6P) were matched along both strain and delay series to the rats of Huskinson et al. (2012), making comparisons between the two studies difficult. The small sample size of the study left inconclusive results regarding whether the effect of $d$-AMP is baseline dependent, 
strain dependent, or housing-type dependent. In addition, the small sample size limited the power of the statistical tests.

As long as AUC is similar across delay series, rats responding in shorter delay series have shorter indifference points. Indeed, in both the present study and in Huskinson et al. (2012), rats responding in the shorter delay series had shorter indifference points at baseline. Differences arose regarding strain because, for Huskinson et al. (2012) LEW rats responded in shorter delay series and had shorter indifference points while in the present study this effect was seen with F344 rats. It may be interesting to manipulate the delay series as an independent variable (provided that no floor or ceiling effects occur).

Another limitation of the present study is that the two groups were nonequivalent. Because the present study was compared to archival data, different experimenters handled and injected the rats. A different experimenter also changed the delay series for each rat. It is possible that the indifference point is affected by the way in which changes in the delay series are made or the way in which rats are handled. However, efforts were made to make paired and singly housed rats as similar as possible. For example, testing began when rats were the same age and similar weight.

Fewer 0 -s probe sessions were conducted in the present study compared to Huskinson et al. (2012) because pair-housed rats were generally slower to reach stability and lever biases occurred for several rats prior to reaching stability. Exposure to fewer 0 -s probe sessions may have affected indifference points as well.

Taken together, the results of the present study indicate that paired housing has differential effects on impulsive choice dependent on baseline impulsivity and strain. Effects of $d$-AMP on impulsive choice depend on these variables as well. The procedure in which 
impulsive choices are measured may also play a role. If the present procedure is replicated, future studies might increase the length of the blackout period used in the beginning of sessions (10 min in the present study) to allow $d$-AMP to metabolize further. This may eliminate disruption of responding during the control block. Future studies might also manipulate cage size and number of animals per cage across the LEW and F344 strains. In addition, research is needed on the role of social dominance in these strains. Specifically, it may be useful to determine if feeding rats while they are together in the home cage or if feeding them individually affects behavior. Ideally, grouping animals by single-housing, social enrichment alone, physical enrichment alone, and a combined social/physical condition would better parse apart effects of housing manipulations. More research is also needed on the cellular levels of dopamine and the extent of dopamine receptor binding in paired and singly housed LEW and F344 rats. Overall, the present study opens many avenues for research examining the neural, behavioral, and environmental mechanisms governing impulsive choice. 


\section{References}

Ainslie, G. (1975). Specious reward: A behavioral theory of impulsiveness and impulse control. Psychological bulletin, 82, 463-496.

Alessi, S.M., \& Petry, N.M. (2003). Pathological gambling severity is associated with impulsivity in a delay discounting procedure. Behavioural Processes, 64, 345-354.

Anderson, K.G., \& Diller, J.W. (2010). Effects of acute and repeated nicotine administration on delay discounting in Lewis and Fischer 344 rats. Behavioural Pharmacology, 21, 754764.

Anderson, K.G., \& Woolverton, W.L. (2005). Effects of clomipramine on self-control choice in Lewis and Fischer 344 rats. Pharmacology Biochemistry, and Behavior, 80, 387-93.

Bardo, M.T., Klebaur, J.E., Valone, J.M., \& Deaton, C. (2001). Environmental enrichment decreases intravenous self-administration of amphetamine in female and male rats. Psychopharmacology, 155, 278-84.

Berton, O., Ramos, A., Chaouloff, F., \& Mormede, P. (1997). Behavioral reactivity to social and nonsocial stimulations: a multivariate analysis of six inbred rat strains. Behavior Genetics, 27, 155-166.

Bickel, W.K., Odum, A.L., \& Madden, G.J. (1999) Impulsivity and cigarette smoking: delay discounting in current, never, and ex-smokers. Psychopharmacology, 146, 447-54.

Burnet, P.W., Mefford, I.N., Smith, C.C., Gold, P.W., \& Sternberg, E.M. (1992). Hippocampal 8 [3H]Hydroxy-2-(di-n-propylamino) tetralin binding site densities, serotonin receptor (5HT1A) messenger ribonucleic acid abundance, and serotonin levels parallel the activity of the hypothalamopituitary-adrenal axis in rat. Journal of Neurochemistry, 59, 10621070. 
Cardinal, R.N., Robbins, T.W., \& Everitt, B.J. (2000). The effects of $d$-amphetamine, chlordiazepoxide, $\alpha$-flupenthixol and behavioural manipulations on choice of signaled and unsignalled delayed reinforcement in rats. Psychopharmacology, 152, 362-75.

Craig, A. R., Maxfield, A. D., Stein, J. S., Renda, C. R., \& Madden, G. J. (2014). Do the adjusting-delay and increasing-delay tasks measure the same construct: delay discounting? Behavioural Pharmacology, 25, 306-315.

deWit, H., Enggasser, J.L., \& Richards, J.B. (2002). Acute administration of $d$-amphetamine decreases impulsivity in healthy volunteers. Neuropsychopharmacology, 28, 813-825.

Diller, J.W., Saunders, B.T., \& Anderson, K.G. (2008). Effects of acute and repeated administration of caffeine on temporal discounting in rats. Pharmacology, Biochemistry, and Behavior, 89, 546-555.

Evenden, J.L., \& Ryan, C.N. (1996). The pharmacology of impulsive behavior in rats: the effects of drugs on response choice with varying delays of reinforcement. Psychopharmacology, $128,161-170$.

Evenden, J.L., \& Ryan, C.N. (1999). The pharmacology of impulsive behavior in rats VI: the effects of ethanol and selective serotonergic drugs on response choice with varying delays of reinforcement. Psychopharmacology, 146, 413-421.

Flores, G., Wood, G. K., Barbeau, D., Quirion, R., \& Srivastava, L. K. (1998). Lewis and Fischer rats: A comparison of dopamine transporter and receptor levels. Brain Research, 814, $34-40$.

Garcia, A., \& Kirkpatrick, K. (2013). Impulsive choice behavior in four strains of rats: Evaluation of possible models of Attention-Deficit/Hyperactivity Disorder. Behavioural Brain Research, 238, 10-22. 
Granon, S., Passetti, F., Thomas, K. L., Dalley, J. W., Everitt, B. J., \& Robbins, T. W. (2000). Enhanced and impaired attentional performance after infusion of D1 dopaminergic receptor agents into rat prefrontal cortex. The Journal of Neuroscience, 20(3), 1208-1215.

Harlan Laboratories, Inc. (2014a). Fischer 344 Inbred Rat. Retrieved from http://www.harlan.com/products_and_services/research_models_and_services/research_ models/fischer_344_inbred_rat.hl.

Harlan Laboratories, Inc. (2014b). Lewis Inbred Rat. Retrieved from http://www.harlan.com/products_and_services/research_models_and_services/research_ models/lewis_inbred_rat.hl.

Ho, M. Y., Wogar, M. A., Bradshaw, C. M., \& Szabadi, E. (1997). Choice between delayed reinforcers: interaction between delay and deprivation level. The Quarterly Journal of Experimental Psychology Section B: Comparative and Physiological Psychology, 50, 193-202.

Hoffmann, L. C., Schütte, S. R. M., Koch, M., \& Schwabe, K. (2009). Effect of “enriched environment" during development on adult rat behavior and response to the dopamine receptor agonist apomorphine. Neuroscience, 158(4), 1589-1598.

Huskinson, S. L., \& Anderson, K. G. (2012). Effects of acute and chronic administration of diazepam on delay discounting in Lewis and Fischer 344 rats. Behavioural Pharmacology, 23, 315-330.

Huskinson, S., Krebs, C.A., \& Anderson, K.G. (2012). Strain differences in delay discounting between Lewis and Fischer 344 rats at baseline and following acute and chronic administration of $d$-amphetamine. Pharmacology, Biochemistry, and Behavior, 101, 403-416. 
Inoue, T., Tsuchiya, K., \& Koyama, T. (1994). Regional changes in dopamine and serotonin activation with various intensity of physical and psychological stress in the rat brain. Pharmacology Biochemistry and Behavior, 49(4), 911-920.

Kirby, K.N., Petry, N.M., \& Bickel, W.K. (1999). Heroin addicts have higher discount rates for delayed rewards than non-drug-using controls. Journal of Experimental Psychology, 128, $78-87$.

Kuczenski, R., \& Segal, D. S. (1997). Effects of methylphenidate on extracellular dopamine, serotonin, and norepinephrine: comparison with amphetamine. Journal of Neurochemistry, 68, 2032-2037.

Madden, G. J., Petry, N. M., Badger, G. J., \& Bickel, W. K. (1997). Impulsive and self-control choices in opioid-dependent patients and non-drug-using control patients: Drug and monetary rewards. Experimental and Clinical Psychopharmacology, 5, 256-262.

Madden, G. J., Smith, N. G., Brewer, A. T., Pinkston, J. W., \& Johnson, P. S. (2008). Steadystate assessment of impulsive choice in Lewis and Fischer 344 Rats: betweencondition delay manipulations. Journal of the Experimental Analysis of Behavior, 90, $333-344$.

Mazur, J.E. An adjusting procedure for studying delayed reinforcement. In: Commons ML, Mazur, J.E., Nevin, J.A., \& Rachlin, H., editors. (1987). Quantitative analysis of behavior: the effect of delay and of intervening events on reinforcement value. Hillside, New Jersey: Lawrence Erlbaum Associates. p. 55-73.

Myerson, J., Green, L., \& Warusawitharana, M. (2001). Area under the curve as a measure of discounting. Journal of the Experimental Analysis of Behavior,76, 235-243.

Perry, J. L., Stairs, D. J., \& Bardo, M. T. (2008). Impulsive choice and environmental 
enrichment: effects of $d$-amphetamine and methylphenidate. Behavioural Brain Research, 193, 48-54.

Petry, N. M. (2001). Pathological gamblers, with and without substance abuse disorders, discount delayed rewards at high rates. Journal of Abnormal Psychology, 110, 482.

Reynolds, B. (2006). A review of delay-discounting research with humans: relations to drug use and gambling. Behavioural Pharmacology, 17, 651-67.

Richards, J. B., Sabol, K. E., \& de Wit, H. (1999). Effects of methamphetamine on the adjusting amount procedure, a model of impulsive behavior in rats. Psychopharmacology, 146, 432-439.

Sagvolden, T., Aase, H., Zeiner, P., \& Berger, D. (1998). Altered reinforcement mechanisms in attention-deficit/hyperactivity disorder. Behavioural Brain Research, 94, 61-71.

Schweitzer, J. B., \& Sulzer-Azaroff, B. (1995). Self-Control in Boys with Attention Deficit Hyperactivity Disorder: Effects of Added Stimulation and Time. Journal of Child Psychology and Psychiatry, 36, 671-686.

Selim, M., \& Bradberry, C. W. (1996). Effect of ethanol on extracellular 5-HT and glutamate in the nucleus accumbens and prefrontal cortex: comparison between the Lewis and Fischer 344 rat strains. Brain Research, 716, 157-164.

Simpson, J., \& Kelly, J. P. (2011). The impact of environmental enrichment in laboratory rats behavioural and neurochemical aspects. Behavioural Brain Research, 222, 246-64.

Solanto, M. V, Abikoff, H., Sonuga-Barke, E., Schachar, R., Logan, G. D., Wigal, T., Hechtman, L., et al. (2001). The ecological validity of delay aversion and response inhibition as measures of impulsivity in AD/HD: a supplement to the NIMH multimodal treatment study of AD/HD. Journal of Abnormal Child Psychology, 29, 215-28. 
Solinas, M., Thiriet, N., Chauvet, C., \& Jaber, M. (2010). Prevention and treatment of drug addiction by environmental enrichment. Progress in Neurobiology, 92, 572-92.

Stein, J. S., Pinkston, J. W., Brewer, A. T., Francisco, M. T., \& Madden, G. J. (2012). Delay discounting in Lewis and Fischer 344 rats: steady-state and rapid-determination adjusting-amount procedures. Journal of the Experimental Analysis of Behavior, 97, 30521.

Stewart, K., Bayne, K. (2004). Environmental enrichment for laboratory animals. In: Reuter JD, editor. Laboratory animal medicine and management. New York: International veterinary information service.

Vijayraghavan, S., Wang, M., Birnbaum, S. G., Williams, G. V., \& Arnsten, A. F. (2007).

Inverted-U dopamine D1 receptor actions on prefrontal neurons engaged in working memory. Nature Neuroscience, 10, 376-384.

Wade TR, de Wit H, Richards JB (2000). Effects of dopaminergic drugs on delayed reward as a measure of impulsive behavior in rats. Psychopharmacology. 150, 90-101.

Weller, R. E., Cook, E. W., Avsar, K. B., \& Cox, J. E. (2008). Obese women show greater delay discounting than healthy-weight women. Appetite, 51, 563-569.

Wilhelm, C. J., \& Mitchell, S. H. (2009). Strain differences in delay discounting using inbred rats. Genes, Brain and Behavior, 8, 426-434.

Winstanley, C.A., Eagle, D.M., \& Robbins, T.W. (2006). Behavioral models of impulsivity in relation to ADHD: translation between clinical and preclinical studies. Clinical Psychology Review, 26, 379-395.

Zeeb, F. D., Wong, A. C., \& Winstanley, C. (2013). Differential effects of environmental enrichment, social-housing, and isolation-rearing on a rat gambling task: Dissociations 
between impulsive action and risky decision-making. Psychopharmacology, 225, 381-95. 
Table 1.

Indifference points at baseline and area under the curve (AUC) at baseline and at each acute administration of d-AMP. Doses of d-AMP are in $\mathrm{mg} / \mathrm{kg}$. The number in parentheses represents the delay series in which the rat listed responded.

\begin{tabular}{|c|c|c|c|c|c|c|c|c|c|}
\hline \multicolumn{3}{|c|}{ Baseline } & \multicolumn{7}{|c|}{ Acute AUC } \\
\hline Subject & IP & AUC & Control & Saline & 0.1 & 0.3 & 0.56 & 1.0 & 1.7 \\
\hline L1S (16) & 4.8 & 0.37 & 0.50 & 0.40 & 0.45 & 0.81 & ----- & 0.67 & omit \\
\hline L2S (16) & 6.6 & 0.47 & 0.44 & 0.38 & 0.58 & 0.65 & --/-- & 0.56 & 0.85 \\
\hline L3S (40) & 9.2 & 0.39 & 0.54 & 0.49 & 0.35 & 0.64 & --/-- & 0.82 & 0.93 \\
\hline L4S (16) & 3.9 & 0.35 & 0.42 & 0.34 & 0.33 & 0.48 & --/-- & 0.50 & 0.76 \\
\hline L5S (40) & 8.5 & 0.34 & 0.26 & 0.21 & 0.33 & 0.28 & 0.65 & 0.42 & $0.75^{a, b}$ \\
\hline L6S (16) & 9.4 & 0.54 & 0.37 & 0.39 & 0.43 & 0.49 & $--/--$ & 0.81 & 0.81 \\
\hline L7S (40) & 12.5 & 0.39 & 0.53 & 0.51 & 0.61 & 0.51 & $0.54^{\mathrm{b}}$ & $0.53^{\mathrm{b}}$ & 0.71 \\
\hline L8S (40) & 25.5 & 0.55 & 0.56 & 0.45 & 0.40 & 0.50 & $--/--$ & $0.60^{\mathrm{b}}$ & 0.89 \\
\hline Mean & 10.0 & 0.43 & 0.45 & 0.39 & 0.44 & 0.54 & 0.60 & 0.61 & 0.81 \\
\hline SEM & 2.4 & 0.03 & 0.04 & 0.03 & 0.04 & 0.06 & 0.05 & 0.05 & 0.03 \\
\hline F1S (60) & 19.7 & 0.38 & 0.49 & 0.37 & 0.39 & 0.45 & 0.36 & $0.19^{b}$ & $0.01^{b}$ \\
\hline F2S (40) & 19.1 & 0.53 & 0.70 & 0.65 & 0.70 & 0.75 & 0.77 & 0.25 & omit \\
\hline F3S (60) & 35.0 & 0.57 & 0.65 & 0.60 & 0.68 & 0.69 & 0.25 & $0.14^{\mathrm{b}}$ & omit \\
\hline F4S (40) & 19.0 & 0.48 & 0.57 & 0.41 & 0.59 & 0.67 & $--/--$ & 0.65 & $0.50^{\mathrm{a}, \mathrm{b}}$ \\
\hline F5S (40) & 8.6 & 0.30 & 0.27 & 0.20 & 0.23 & 0.31 & $--/--$ & 0.60 & 0.52 \\
\hline F6S (40) & 16.8 & 0.48 & 0.41 & 0.33 & 0.49 & 0.46 & $--/--$ & 0.45 & 0.45 \\
\hline F7S (60) & 36.7 & 0.59 & 0.34 & 0.33 & 0.23 & 0.37 & 0.30 & 0.20 & $0.06^{\mathrm{b}}$ \\
\hline F8S (40) & 9.4 & 0.32 & 0.42 & 0.37 & 0.34 & 0.47 & 0.52 & $0.49^{b}$ & $0.05^{\mathrm{b}}$ \\
\hline Mean & 20.5 & 0.46 & 0.48 & 0.41 & 0.46 & 0.52 & 0.44 & 0.37 & 0.27 \\
\hline SEM & 3.7 & 0.04 & 0.05 & 0.05 & 0.07 & 0.06 & 0.09 & 0.07 & 0.10 \\
\hline L3P (60) & 11.0 & 0.63 & 0.47 & 0.44 & 0.46 & 0.54 & 每/-- & $0.42^{\mathrm{b}}$ & omit \\
\hline L4P (60) & 10.5 & 0.52 & 0.36 & 0.31 & 0.42 & 0.45 & 0.53 & $0.48^{\mathrm{b}}$ & 0.42 \\
\hline L5P (16) & 2.7 & 0.22 & 0.22 & $0.34^{\mathrm{b}}$ & $0.24^{\mathrm{b}}$ & $0.43^{\mathrm{b}}$ & $--/--$ & $0.67^{\mathrm{b}}$ & $0.88^{\mathrm{b}}$ \\
\hline L6P (40) & 11.6 & 0.33 & 0.19 & 0.22 & 0.21 & 0.36 & --/-- & $0.28^{\mathrm{b}}$ & $0.06^{\mathrm{b}}$ \\
\hline L7P (60) & 54.6 & 0.66 & 0.39 & 0.48 & 0.42 & $0.55^{\mathrm{b}}$ & $--/--$ & $0.50^{\mathrm{b}}$ & 0.82 \\
\hline L8P (40) & 22.2 & 0.37 & 0.26 & 0.26 & 0.15 & 0.26 & $--/--$ & $0.22^{\mathrm{b}}$ & $0.29^{b}$ \\
\hline Mean & 18.8 & 0.45 & 0.32 & 0.34 & 0.32 & 0.43 & 0.53 & 0.43 & 0.49 \\
\hline SEM & 7.6 & 0.07 & 0.04 & 0.04 & 0.05 & 0.04 & $--/--$ & 0.07 & 0.14 \\
\hline F1P (40) & 10.4 & 0.53 & 0.51 & 0.53 & 0.64 & 0.52 & 0.46 & 0.68 & omit \\
\hline F2P (16) & 6.8 & 0.47 & 0.23 & 0.17 & 0.21 & 0.53 & 0.43 & 0.68 & 0.71 \\
\hline F3P (16) & 6.4 & 0.44 & 0.31 & 0.21 & 0.27 & 0.50 & $--/--$ & 0.50 & $0.62^{\mathrm{b}}$ \\
\hline F4P (16) & 2.8 & 0.29 & 0.34 & 0.22 & 0.29 & 0.60 & $0.37^{\mathrm{b}}$ & $0.03^{\mathrm{b}}$ & omit \\
\hline F5P (16) & 3.9 & 0.39 & 0.31 & 0.26 & 0.27 & 0.63 & $--/--$ & $0.56^{\mathrm{b}}$ & 0.95 \\
\hline F6P (40) & 6.2 & 0.45 & 0.39 & 0.33 & 0.35 & 0.45 & $--/--$ & $0.28^{\mathrm{b}}$ & omit \\
\hline Mean & 6.1 & 0.43 & 0.35 & 0.29 & 0.34 & 0.54 & 0.42 & 0.45 & 0.76 \\
\hline SEM & 1.0 & 0.03 & 0.04 & 0.05 & 0.06 & 0.03 & 0.03 & 0.10 & 0.10 \\
\hline
\end{tabular}

a Based on only one determination.

b Larger-reinforcer choice was less than $75 \%$ in the first delay block (0s) 


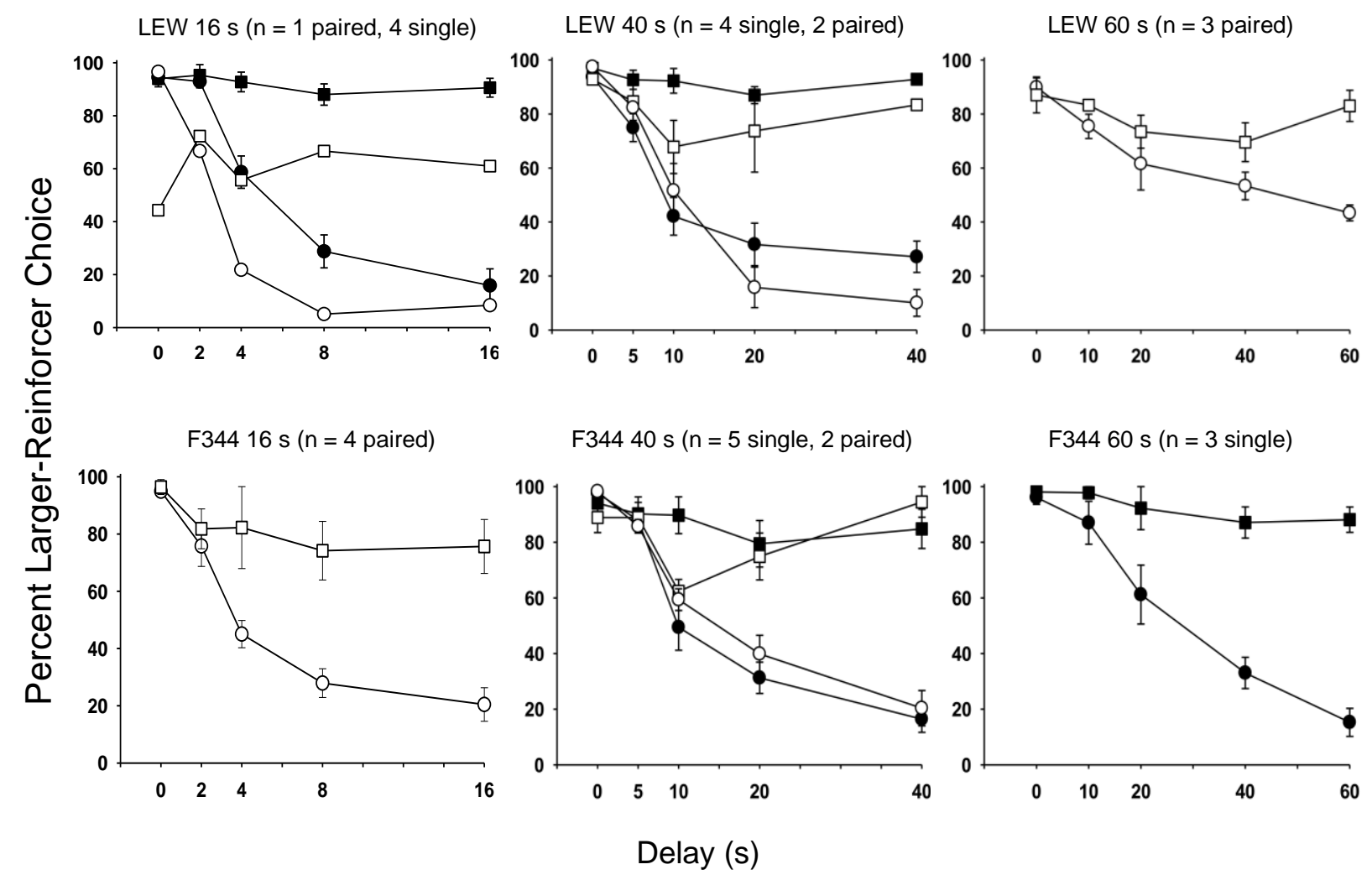

Figure 1. Average percent larger-reinforcer choice as a function of delay for the last 10 sessions of baseline in singly housed rats $(\bullet)$ and pair-housed rats $(O)$. Average percent larger reinforcer choice during the last 0 -s probe sessions before drug administration in singly housed rats ( $\mathbf{\square})$ and pair-housed rats $(\square)$ is shown in the same panels with baseline data. 


\section{Indifference Points}

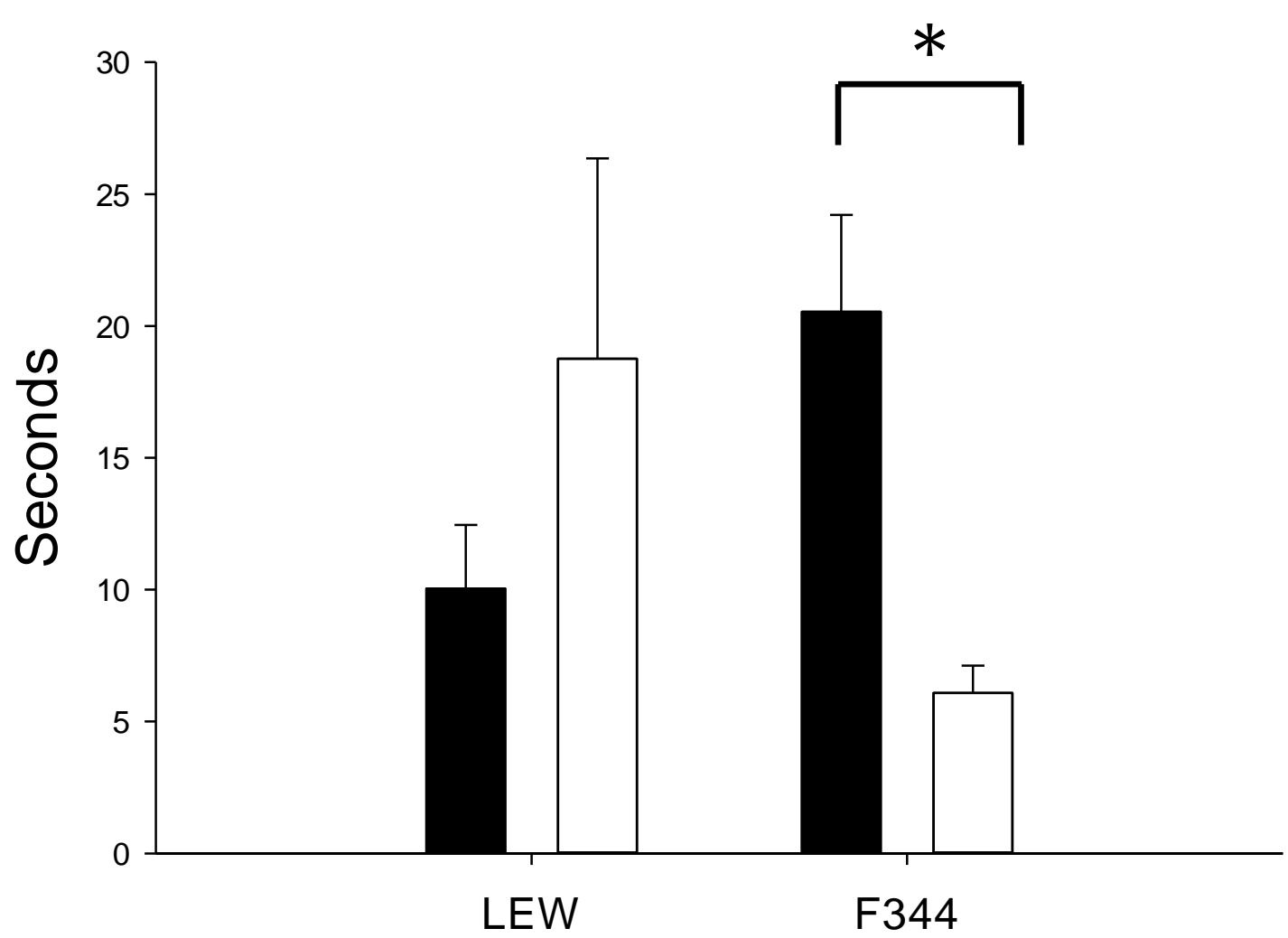

Figure 2. Indifference points for paired and singly housed rats from the last 10 sessions of the baseline condition. The asterisk represents a statistically significant difference $(\mathrm{p}<.05)$. 


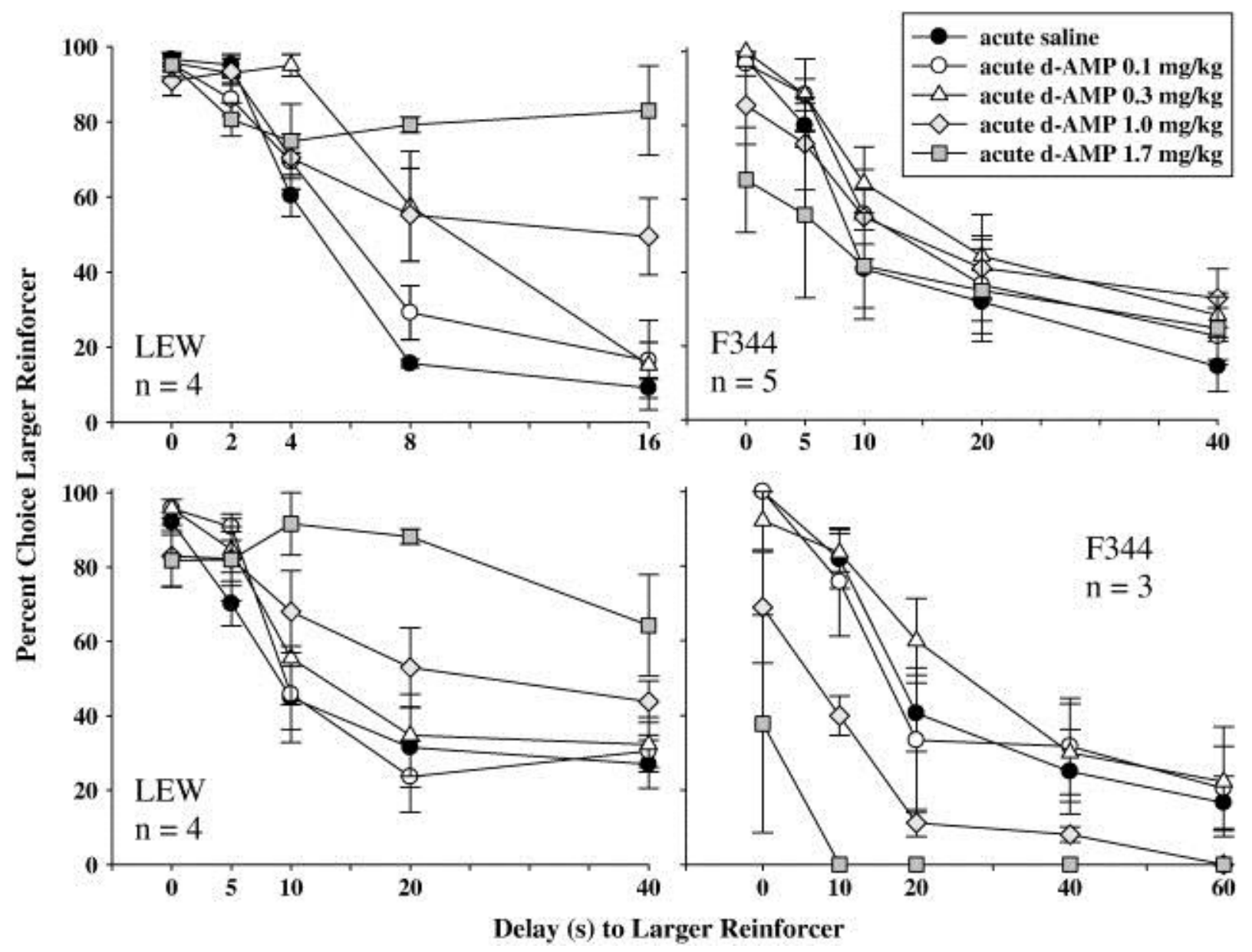

Figure 3a. Percent larger-reinforcer choice as a function of delay for singly housed rats. Filled circles represent saline and open symbols represent doses of $d$-AMP. Rats are grouped by strain and delay series. Error bars represent standard error of the mean. (Copied directly from Huskinson et al., 2012.) 

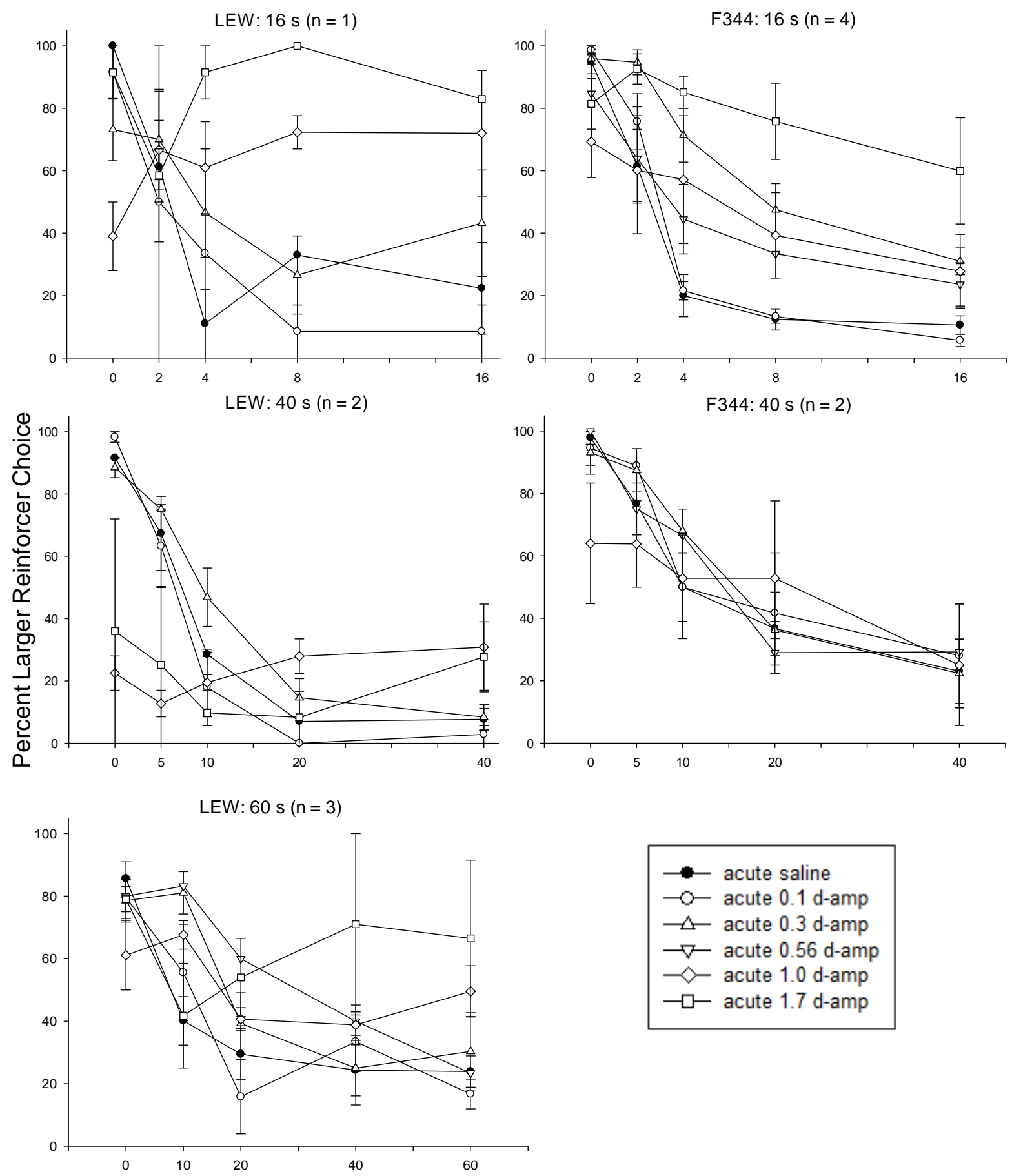

Delay (s)

Figure $3 b$. Percent larger-reinforcer choice as a function of delay for pair-housed rats. Filled circles represent saline and unfilled symbols represent doses of $d$-AMP. Rats are grouped by strain and delay series. 
Singly Housed
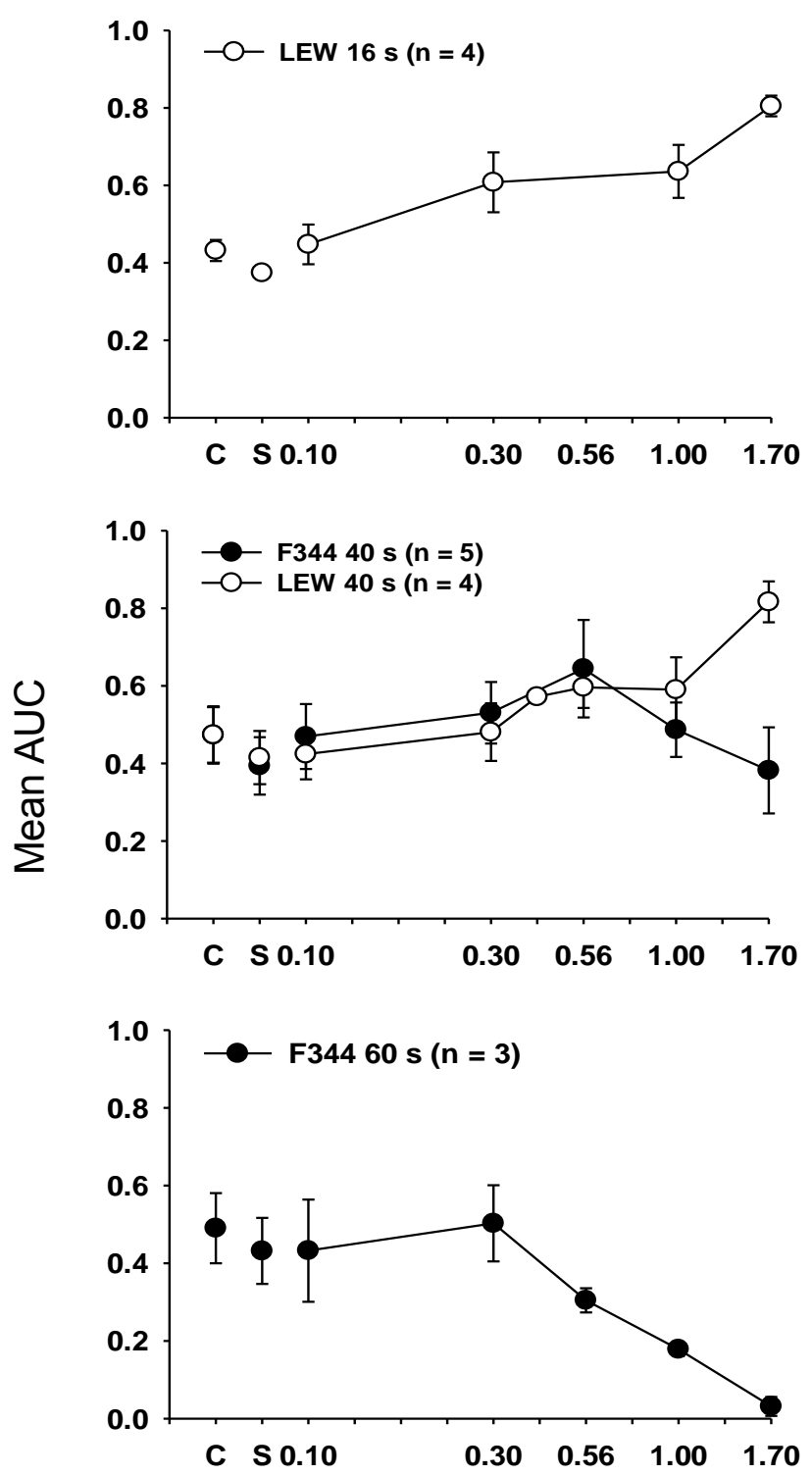

Pair-housed
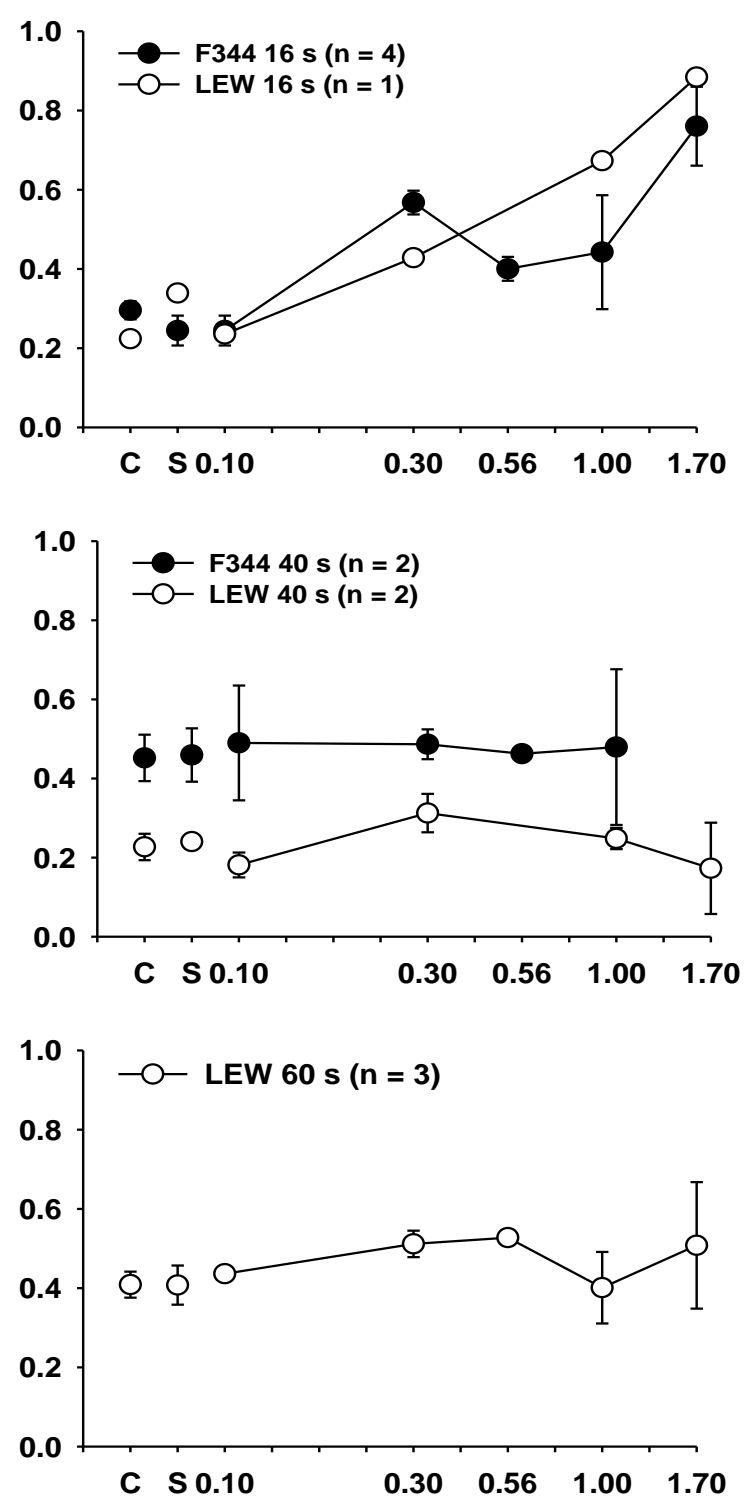

d-AMP Dose $(\mathrm{mg} / \mathrm{kg})$

Figure 4. AUC as a function of dose for the singly housed rats of Huskinson et al. (2012; left) and the pair-housed rats of the present study (right). Groups are divided based on the delay series in which they responded in. "C" represents control sessions in which no injection was given, and "S" represents sessions in which saline was administered. (Note, for pair-housed F344 rats, responding was suppressed at the $1.7 \mathrm{mg} / \mathrm{kg}$ dose. 


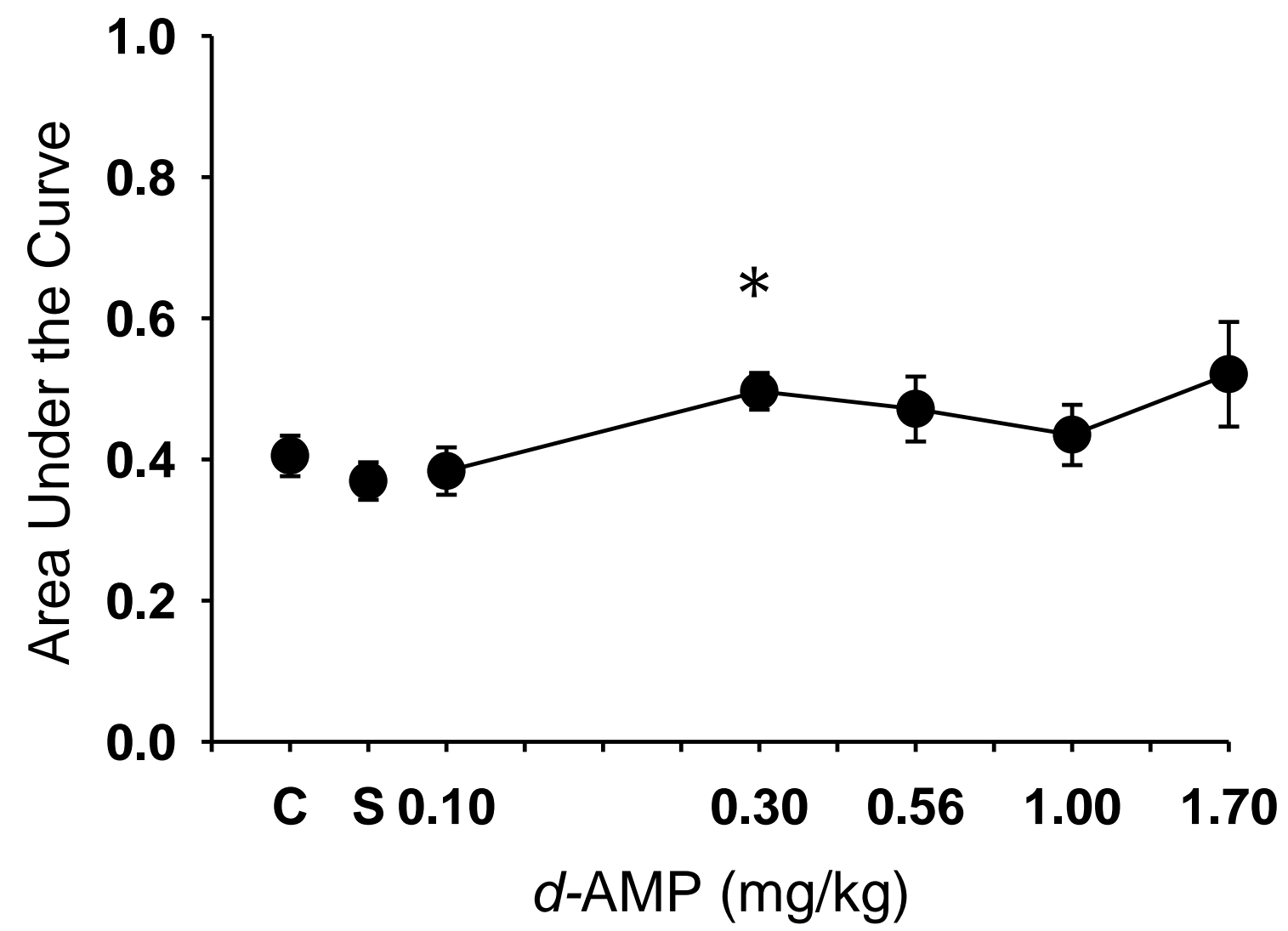

Figure 5. AUC as a function of dose collapsed across singly housed and pair-housed rats. "C" represents control sessions in which no injection was given, and " $\mathrm{S}$ " represents sessions in which saline was administered. The asterisk indicates that the dose of $0.3 \mathrm{mg} / \mathrm{kg}$ was significantly different from saline. 